\title{
Efecto del uso de hidrógeno en la potencia y rendimiento de un motor de combustión interna
}

\section{(Effect of using hydrogen in the power and performance of an internal combustion engine)}

\author{
Edwin Tamayo ${ }^{1}$, Carlos Rosales ${ }^{1}$, Alex Guzmán ${ }^{1}$, Paúl Pazmiño ${ }^{2}$
}

\begin{abstract}
Resumen:
En el presente estudio se analizaron los parámetros del funcionamiento real de un motor de combustión interna ciclo Otto, que utiliza como combustible hidrógeno más gasolina; se determinaron dos ecuaciones estequiométricas. En la primera ecuación, los reactivos son el octano y el aire, en la segunda ecuación se adicionó la cantidad real de 3.86 moles de $\mathrm{H}_{2}$ obtenidas de una celda de hidrógeno. A partir de las ecuaciones químicas se determinaron dos sistemas de ecuaciones para obtener el consumo y la potencia, manteniendo las condiciones a la altura de Quito de $2850 \mathrm{msnm}, 72.794 \mathrm{kPa}$ de presión atmosférica y $300 \mathrm{~K}$ de temperatura ambiente. Se utilizó un motor monocilindro alimentado con hidrógeno más gasolina, y se obtuvieron datos reales de potencia del motor con las mezclas aire-gasolina e hidrógenogasolina. La potencia teórica sin $\mathrm{H}_{2}$ fue $3.91 \mathrm{HP}$ y con $\mathrm{H}_{2}$ de $5.41 \mathrm{HP}$, con un aumento del $27.1 \%$; la potencia real es $3.78 \mathrm{HP} \sin \mathrm{H}_{2}$ y $4.66 \mathrm{HP}_{\text {con }} \mathrm{H}_{2}$, la cual se incrementó en un $16.7 \%$. El consumo de combustible teórico es $401.61 \mathrm{~g} / \mathrm{kWh}$ y con adición de $\mathrm{H}_{2}$ es menor a 373.52 $\mathrm{g} / \mathrm{kWh}$; el consumo real que indica el fabricante es $395 \mathrm{~g} / \mathrm{kWh}$.
\end{abstract}

Palabras clave: hidrógeno; estequiometrías; Otto; potencia

\begin{abstract}
:
This study analyzed the real working parameters of an Otto cycle internal combustion engine, using as fuel hydrogen plus gasoline. Two stoichiometric equations were determined. In the first equation, the reagents are octane and air, in the second equation was added the quantity of 3.86 $\mathrm{H}_{2}$ moles obtained from a hydrogen cell. Two sets of equations, for consumption and power, were determined from the chemical equations, working at the conditions of Quito: altitude $2850 \mathrm{msnm}, 72.794 \mathrm{kPa}$ of atmospheric pressure and $300 \mathrm{~K}$ of temperature. A single cylinder engine powered with hydrogen plus gasoline was used for getting real data of engine power, using mixtures of air-gasoline and hydrogen; the theoretical power without $\mathrm{H}_{2}$ was 3.91 $\mathrm{HP}$ and with $\mathrm{H}_{2} 5.41 \mathrm{HP}$, it increased $27.1 \%$, the real power is $3.78 \mathrm{HP}$ without $\mathrm{H}_{2}$ and $4.66 \mathrm{HP}$ with $\mathrm{H}_{2}$, it increased $16.7 \%$. Theoretical fuel consumption is $401.61 \mathrm{~g} / \mathrm{kWh}$ and addition of $\mathrm{H}_{2}$ is less to $373.52 \mathrm{~g} / \mathrm{kWh}$, the actual consumption that indicates the manufacturer is $395 \mathrm{~g} / \mathrm{kWh}$.
\end{abstract}

Keywords: hydrogen; stoichiometric; Otto; power

\footnotetext{
${ }^{1}$ Universidad Tecnológica Equinoccial, Quito - Ecuador ( \{edwin.tamayo, carlos.rosales, alex.guzman\} @ute.edu.ec)

${ }^{2}$ Universidad Tecnológica Equinoccial, Quito - Ecuador (paulpaz512g@gmail.com.ec)
} 


\section{Introducción}

Las tecnologías de los autos están continuamente en renovación, tanto en diseño cuanto en construcción de motores de combustión interna, con lo cual se ha elevado el rendimiento relacionado al incremento de potencia sin que dé como resultado un consumo elevado de combustible, ya que a mayor consumo mayor producción de emisiones de gases contaminantes son emanados a la atmósfera.

Con la intención de aportar con un estudio y alternativa tecnológica eficiente para disminuir las emisiones contaminantes y obtener un mejor rendimiento en la potencia de los motores de combustión interna, se ha optado por el uso del hidrógeno como combustible mediante un sistema de generación e inyección del mismo en el motor durante el proceso de admisión del motor, además de ser un estudio base para desarrollo tecnológico en ciudades de altura (respecto al nivel del mar) como es el caso de la ciudad de Quito, la misma que está a una altura promedio de 2850 metros.

Los problemas derivados de la actual política energética son aquellos relacionados con la contaminación. Algunos de los contaminantes generados en la combustión de los hidrocarburos son responsables de numerosas enfermedades pulmonares (Fàbrega, 2009), mientras que otros, como el monóxido de carbono en altas concentraciones, aumentan la probabilidad de sufrir un infarto (Bennaceur et al., 2005). Estos contaminantes no solo afectan directamente al ser humano, sino que también lo están haciendo de forma indirecta gracias al temido efecto invernadero, del que ya se están empezando a notar sus efectos en el clima global (Velástegui et al., 2015).

Por numerosas razones, el punto de introducción para la energía con base en el hidrógeno es el sector del transporte. Los consumidores pagan considerablemente mucho más por la energía utilizada en el transporte que por la electricidad o el gas empleado para fines domésticos (esta relación podría ser, conservadoramente, hasta de 8 a 1 para una familia de clase media). Más aún, el sector del transporte tiene también un gran potencial de ganancias por eficiencia de combustible. En efecto, la eficiencia de los automóviles modernos es de alrededor del 13 por ciento durante el ciclo de manejo urbano, en tanto que los vehículos a hidrógeno, ya sean híbridoeléctricos o de celdas de combustible, podrían alcanzar eficiencias del orden de entre 35 y 45 por ciento. Los vehículos impulsados por hidrógeno o con mezclas gasolina - hidrógeno pueden cumplir con la demanda creciente de bajas o cero emisiones (Gutiérrez, 2005).

Un prototipo funcional de vehículo dual (hidrógeno/gasolina) fue desarrollado en base a un Volkwagen Polo 1.4, el funcionamiento dual (hidrógeno/gasolina) permite a un vehículo funcionar con hidrógeno en recorridos urbanos, reduciendo así la contaminación. Para rutas interurbanas y mayores distancias se puede hacer uso de la gasolina gracias a su mayor potencia y autonomía. Esta tecnología sería aplicable de forma inmediata y a un coste razonable, mientras se va 
desarrollando el vehículo de pila de combustible, y podría ayudar al despliegue de la infraestructura del hidrógeno (Sáinz, 2014).

La adición de hidrógeno con el uso de gasolina extra genera mejores resultados en comparación a un motor funcionando con gasolina súper; incrementando la potencia, torque y reduciendo en su mayoría las emisiones contaminantes para ambos casos, al igual que el consumo para el caso de gasolina extra. Las emisiones contaminantes analizadas tuvieron una disminución de $\mathrm{CO}_{2}$ con $4.43 \%$, los $\mathrm{HC}$ en $1.76 \%$, los $\mathrm{NOx}$ con $11.82 \%$, y un aumento en $\mathrm{CO}$ con $1.43 \%$ y el $\mathrm{O}_{2}$ en $6.03 \%$ en relación con los análisis con gasolina súper (Velástegui et al, 2015).

El hidrógeno $\left(\mathrm{H}_{2}\right)$ es un gas incoloro, inodoro e insípido que es 14.4 veces más ligero que el aire. Su masa atómica y molecular son $1.007940 \mathrm{~kg} / \mathrm{kmol}$ y $2.01588 \mathrm{~kg} / \mathrm{kmol}$ respectivamente. Se encuentra en estado gaseoso a 25ํㅡ y 1 atm (101.325 kPa) y su temperatura de fusión es de $-259.2^{\circ} \mathrm{C}$. La temperatura de ebullición de esta sustancia es de $-252.77^{\circ} \mathrm{C}$. La constante específica del gas (que equivale a la constante universal de los gases entre el peso molecular del hidrógeno) es $4124.3 \mathrm{~J} / \mathrm{kgK}$ y su constante de difusión es $0.61 \mathrm{~cm}^{2} / \mathrm{s}$. El hidrógeno gaseoso es muy inflamable y arde en concentraciones muy bajas en aire $\left(4 \%\right.$ de $\left.\mathrm{H}_{2}\right)$. La entalpía de combustión del hidrógeno es -286 kJ/mol (Familiar, 2011).

Los sistemas duales consisten en usar una cantidad relativamente pequeña de hidrógeno para modificar las características de la combustión y de las emisiones, o bien usar cantidades mayores de hidrógeno como fuente principal de energía y controlar el encendido mediante la inyección de otro combustible dentro de la cámara de combustión. El hidrógeno tiene unas ventajas destacables comparadas con las de otros combustibles alternativos ya que este gas no contiene carbono, aspecto que resulta en una casi total eliminación de las emisiones de $\mathrm{CO}, \mathrm{CO}_{2}$ y de hidrocarburos no combustionados (HC) (Familiar, 2011).

En el caso que el motor utilizado fuera de encendido mediante chispa, se podría utilizar hidrógeno como único combustible (single-fuel), ya que la ignición sería producida mediante la bujía. Usando hidrógeno como único combustible se conseguiría una potencia similar a un motor de gasolina, con un rendimiento mayor. Los inconvenientes de un motor de chispa con hidrógeno derivan en el retorno de la llama, la preignición y las emisiones de óxidos de nitrógeno. La preignición suele darse cuando la mezcla de hidrógeno y aire se enciende incluso antes de la generación del arco eléctrico en la bujía, produciéndose una combustión ineficiente y un funcionamiento anormal. Las fuentes de dicha ignición a destiempo suelen ser los puntos de mayor temperatura en el interior de la cámara de combustión, los gases calientes en el interior de dicha cámara y en especial la alta temperatura en la válvula de escape. Cuando dicho prencendido se da cerca de la válvula de aspiración, la llama viaja hacia atrás por las tuberías de alimentación resultando en detonaciones. Estos problemas pueden solucionarse en gran medida con la inyección del hidrógeno mediante un inyector, en lugar de usar el método de carburación (Casaravilla, 2012). 
En la electrólisis del agua, la electricidad se utiliza para descomponer el agua en sus componentes elementales: hidrógeno y oxígeno. El dispositivo que se utiliza para llevar a cabo la electrólisis del agua es una cuba electrolítica, que consta de una serie de células, cada una con un electrodo positivo y otro negativo. Los electrodos se sumergen en un electrolito (agua eléctricamente conductora, agregando los iones del hidrógeno, generalmente bajo la forma de hidróxido alcalino de potasio, $\mathrm{KOH}$ ) para facilitar la migración de los iones.

El índice de generación de hidrógeno se relaciona con la densidad de corriente (cantidad de corriente dividida por el área del electrodo, medida en amperios por área). Generalmente cuanto más alta es la densidad de corriente, mayor es el voltaje requerido de la fuente de alimentación, y por tanto mayor será el costo de energía por unidad de hidrógeno. No obstante, valores elevados de voltaje disminuyen el tamaño de la cuba electrolítica y por lo tanto dan lugar a un coste menor de la instalación. Las cubas electrolíticas avanzadas son fiables, con rendimientos energéticos entre $65 \%$ y $80 \%$, funcionan con densidades de corriente en torno a $2000 \mathrm{~A} / \mathrm{m}^{2}$. La cantidad de energía eléctrica requerida para la electrólisis del agua puede compensarse agregando energía térmica a la reacción. La cantidad mínima de voltaje requerida para descomponer el agua es de $1.23 \mathrm{~V}$ a $25^{\circ} \mathrm{C}$. A este voltaje, la reacción requiere energía térmica del exterior para producirse. A $1.47 \mathrm{~V}$ y la misma temperatura que en el caso anterior, no se requiere ninguna cantidad de calor aportada (Fàbrega., 2009).

\section{Metodología}

Para el desarrollo de la investigación se utilizó un banco dinamómetro, con un motor generador accionado por un motor de combustión interna monocilindro. En la Tabla 1 se indican las especificaciones técnicas de los dos elementos.

Tabla 1. Características del motor de combustión interna monocilindro y del motor generador.

\begin{tabular}{|c|c|c|}
\hline \multirow{10}{*}{$\begin{array}{c}\text { MOTOR } \\
\text { MONOCILINDRO }\end{array}$} & Modelo & TEMCO 160 \\
\hline & Tipo & Cilindro simple 4 tiempos OHV \\
\hline & Cilindraje & $163 \mathrm{cc}$ \\
\hline & Relación de compresión & $8.5: 1$ \\
\hline & Frecuencia de giro (RPM) & 3600 \\
\hline & Torque máximo & $10 / 2500$ \\
\hline & Consumo de combustible $(\mathrm{g} / \mathrm{kW} \cdot \mathrm{h})$ & 395 \\
\hline & $\begin{array}{l}\text { Sistema de ignición } \\
\end{array}$ & Magneto con transistor \\
\hline & Capacidad de aceite (L) & 0.6 \\
\hline & Capacidad de gasolina en (L) & 3.6 \\
\hline \multirow{5}{*}{$\begin{array}{c}\text { MOTOR } \\
\text { GENERADOR }\end{array}$} & Frecuencia & $60 \mathrm{~Hz}$ \\
\hline & Potencia de salida continua & $2700 \mathrm{~W}$ \\
\hline & Potencia de salida máxima & $3000 \mathrm{~W}$ \\
\hline & Regulación de voltaje & Regulado por condensador \\
\hline & Frecuencia de giro / peso & 3000 RPM / $38 \mathrm{~kg}$ \\
\hline
\end{tabular}

La generación del hidrógeno se realizó a volumen constante con 12V, la electrólisis se produjo utilizando hidróxido de sodio al $50 \%$ en volumen, con un burbujeador como componente de seguridad del sistema ante posibles explosiones. En la Figura 1 se indican los elementos 
principales del generador de hidrógeno instalado sobre el banco dinamómetro para medición de potencia.
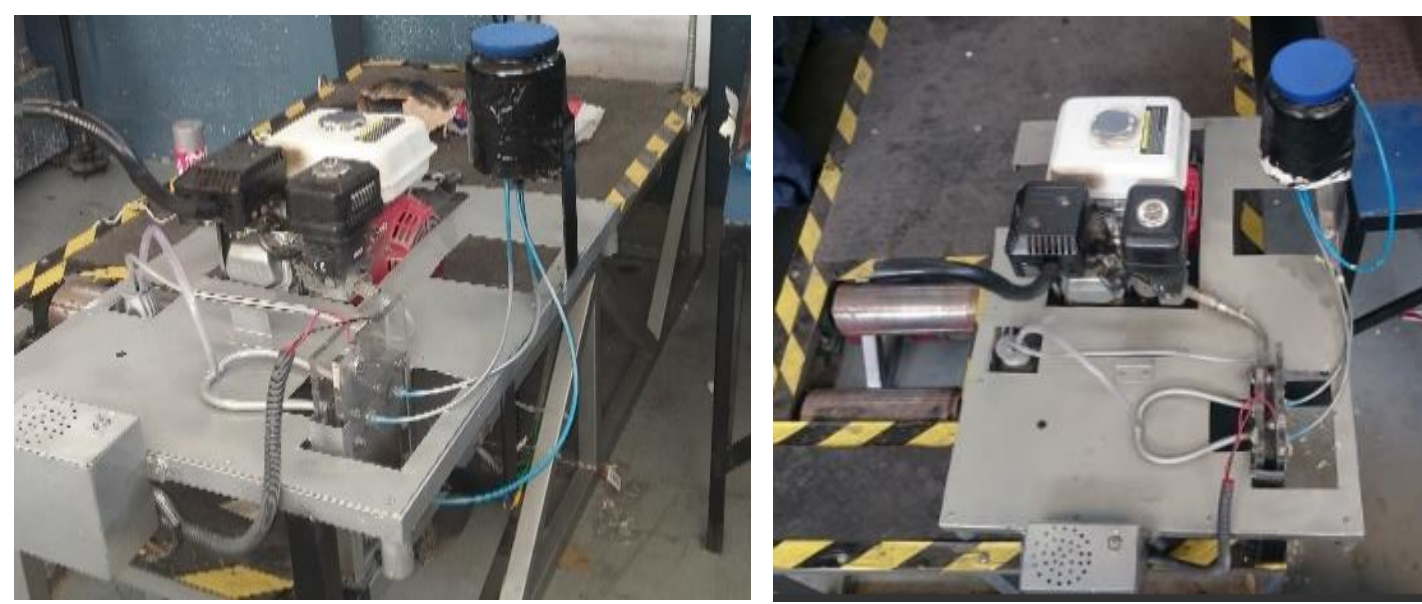

Figura 1. Generador de hidrógeno sobre el banco dinamómetro para medición de potencia.

Se plantearon 2 ecuaciones estequiométricas, una para el cálculo con la mezcla gasolina-aire y gasolina-hidrógeno. Se desarrollaron 6 sistemas de ecuaciones termodinámicas teóricas del ciclo Otto para la obtención de los resultados de rendimiento, consumo y potencia. Tres sistemas para el cálculo con la mezcla gasolina-aire y 3 para gasolina-hidrógeno, los resultados se compararon con los valores reales obtenidos en el freno dinamómetro, para así determinar con más relevancia lo sucedido respecto a la potencia en la ciudad de Quito ubicada a $2850 \mathrm{msnm}$, a presión atmosférica de $72.795 \mathrm{kPa}$ y a una temperatura de $300 \mathrm{~K}$.

La investigación se ejecutó en tres etapas, en la primera etapa se establecieron 2 ecuaciones estequiométricas de mezcla; con la primera ecuación se calcula usando gasolina más aire. En los reactivos se considera al octano como el componente que tiene un gran poder calorífico, el aire es dividido en dos compuestos principales, oxígeno y nitrógeno, y se consideran productos al dióxido de carbono, el agua y nitrógeno, como se muestra en la Ecuación 1.

$$
\mathrm{C}_{8} \mathrm{H}_{18}+\frac{25}{2}\left(\mathrm{O}_{2}+(3.76) \mathrm{N}_{2}\right) \rightarrow 8 \mathrm{CO}_{2}+9 \mathrm{H}_{2} \mathrm{O}+\frac{25}{2}(3.76) \mathrm{N}_{2}
$$

La segunda ecuación estequiométrica se obtiene al adicionar hidrógeno a la gasolina; para los reactivos se consideró el mismo valor de octano, del oxígeno y nitrógeno; al aumentar el hidrógeno en los productos no se produce ningún compuesto adicional, pero al combustionarse aumenta el valor de agua y de nitrógeno.

$$
\mathrm{C}_{8} \mathrm{H}_{18}+3.834 \mathrm{H}_{2}+28.834\left(\mathrm{O}_{2}+(3.76) \mathrm{N}_{2} \rightarrow 8 \mathrm{CO}_{2}+\frac{25.668}{2} \mathrm{H}_{2} \mathrm{O}+28.834(3.76) \mathrm{N}_{2}\right.
$$


En la segunda etapa se utilizaron 3 sistemas de ecuaciones termodinámicas para la mezcla gasolina más aire y 3 sistemas de ecuaciones para gasolina más hidrógeno, cada sistema parte de una ecuación matriz del rendimiento volumétrico, potencia y consumo específico, como se indica en las ecuaciones 3, 4 y 5 respectivamente (Cengel, 2012).

\section{Rendimiento volumétrico:}

Para el cálculo del rendimiento volumétrico del motor se utiliza la expresión siguiente:

$$
\mathrm{n}_{\mathrm{v}}=\left(\frac{\mathrm{r}}{\mathrm{r}-1}\right)\left(\frac{\mathrm{P}_{1}}{\mathrm{P}_{\mathrm{a}}}\right)\left[\frac{\mathrm{T}_{1}}{\mathrm{~T}_{\mathrm{a}}\left(1+\gamma_{\mathrm{r}}\right)}\right]
$$

Donde:

$\mathrm{n}_{\mathrm{v}}$ : Rendimiento volumétrico

r: Es la relación de compresión

$\mathrm{P}_{\mathrm{a}}$ : Es la presión atmosférica

$\mathrm{P}_{1}$ : Es la presión de admisión

$\mathrm{T}_{\mathrm{a}}$ : Es la temperatura ambiente

$\mathrm{T}_{1}$ : Es la temperatura de admisión

$\gamma_{\mathrm{r}}$ : Es el coeficiente de gases residuales

\section{Potencia teórica}

Para determinar la potencia teórica del motor se aplica la siguiente ecuación:

$$
\mathrm{N}_{\mathrm{t}}=\mathrm{z}\left(\mathrm{PCI}\left[\frac{\mathrm{kJ}}{\mathrm{kg}}\right] * \mathrm{~m}_{\mathrm{f}}[\mathrm{kg}]\right) \text { A }\left[\frac{\mathrm{kPa}}{\mathrm{kJ}}\right]\left[\frac{1.36 \mathrm{CV}}{1 \mathrm{kPa}}\right]\left[\frac{0.735 \mathrm{~kW}}{1 \mathrm{CV}}\right] \mathrm{n}_{\mathrm{t}}\left(\frac{\mathrm{n}}{2 * 60}\right)
$$

Donde:

$\mathrm{N}_{\mathrm{t}}$ : Es la potencia teórica

z: Es el número de cilindros

PCI: Poder calorífico inferior

$\mathrm{m}_{\mathrm{f}}$ : Flujo másico del combustible

A: Constante de transformación de calor en trabajo mecánico 1.000152754

$\mathrm{n}_{\mathrm{t}}$ : Rendimiento térmico

$\mathrm{n}$ : Frecuencia

\section{Consumo específico}

El consumo específico de combustible se determinó con la relación:

$$
\mathrm{c}_{\mathrm{e}}=\frac{\dot{\mathrm{m}}_{\mathrm{f}}\left[\frac{\mathrm{g}}{\mathrm{s}}\right] * 3600 \mathrm{~s} * \mathrm{~V}_{\mathrm{T}}\left[\mathrm{m}^{3}\right] \mathrm{n}_{\mathrm{i}} * \mathrm{n}_{\mathrm{m}}}{\mathrm{N}_{\mathrm{t}}[\mathrm{kW}] * \mathrm{n}_{\mathrm{v}}}
$$

Donde:

$\mathrm{c}_{\mathrm{e}}$ : Consumo específico 
$\mathrm{m}_{\mathrm{f}}$ : Flujo másico de combustible

$\mathrm{V}_{\mathrm{T}}$ : Volumen total motor

$\mathrm{n}_{\mathrm{i}}$ : Rendimiento Interno

$\mathrm{n}_{\mathrm{m}}$ : Rendimiento mecánico

$\mathrm{N}_{\mathrm{t}}$ : Potencia teórica

$\mathrm{n}_{\mathrm{v}}$ : Rendimiento Volumétrico

\section{Resultados y Discusión}

Con las 2 ecuaciones estequiométricas y los 6 sistemas de ecuaciones se calculó el rendimiento volumétrico, la potencia y el consumo de combustible, y se obtuvieron los resultados teóricos para la mezcla gasolina más aire y gasolina más hidrógeno, los cuales se indican en la Tabla 2.

Tabla 2. Resultados en la mezcla gasolina más aire y gasolina más hidrógeno.

\begin{tabular}{|c|c|c|c|}
\hline Mezcla & Rendimiento volumétrico & Potencia & Consumo específico \\
\hline Gasolina + Aire & 0.6205 & $3.91 \mathrm{HP}$ & 401.61 \\
\hline Gasolina + Hidrógeno & 0.6205 & $5.40 \mathrm{HP}$ & 373.72 \\
\hline
\end{tabular}

La tercera etapa fue la obtención de los valores reales del motor monocilindro del primer banco de pruebas acoplado al dinamómetro, para lo cual se determinó la variación de la potencia respecto a las revoluciones en la mezcla gasolina más aire como se muestra en la Tabla 3.

Tabla 3. Resultados de pruebas del motor monocilindro sin ingreso de $\mathrm{H}_{2}$ en el dinamómetro.

\begin{tabular}{|c|c|c|c|c|c|}
\hline $\begin{array}{c}\text { Nivel de } \\
\text { carga }\end{array}$ & RPM & $\begin{array}{c}\text { Voltaje } \\
\text { (V) }\end{array}$ & $\begin{array}{c}\text { Amperaje } \\
(\mathbf{A})\end{array}$ & $\begin{array}{c}\text { Potencia } \\
\text { (A.V=W) }\end{array}$ & $\begin{array}{c}\text { Potencia } \\
\text { (HP) }\end{array}$ \\
\hline 1 & 3600 & 90 & 2 & 180 & 1.091 \\
\hline 2 & 3500 & 80 & 3 & 240 & 1.454 \\
\hline 3 & 3400 & 71 & 7 & 497 & 3.011 \\
\hline 4 & 3300 & 60 & 9 & 540 & 3.272 \\
\hline 5 & 3200 & 52 & 12 & 624 & 3.781 \\
\hline 6 & 3000 & 41 & 13 & 533 & 3.229 \\
\hline 7 & 2700 & 35 & 15 & 525 & 3.181 \\
\hline 8 & 2400 & 31 & 16 & 496 & 3.005 \\
\hline
\end{tabular}

Se observa que la potencia máxima obtenida es de 3.78 HP a 3200 rpm con un nivel de carga 5, lo cual significa que a partir de este punto, si las revoluciones aumentan la potencia irá descendiendo.

La curva de la relación potencia respecto a la frecuencia de giro (rpm) con el nivel de carga que generaba el motor generador del banco freno dinamómetro se indica en la Figura 2. 


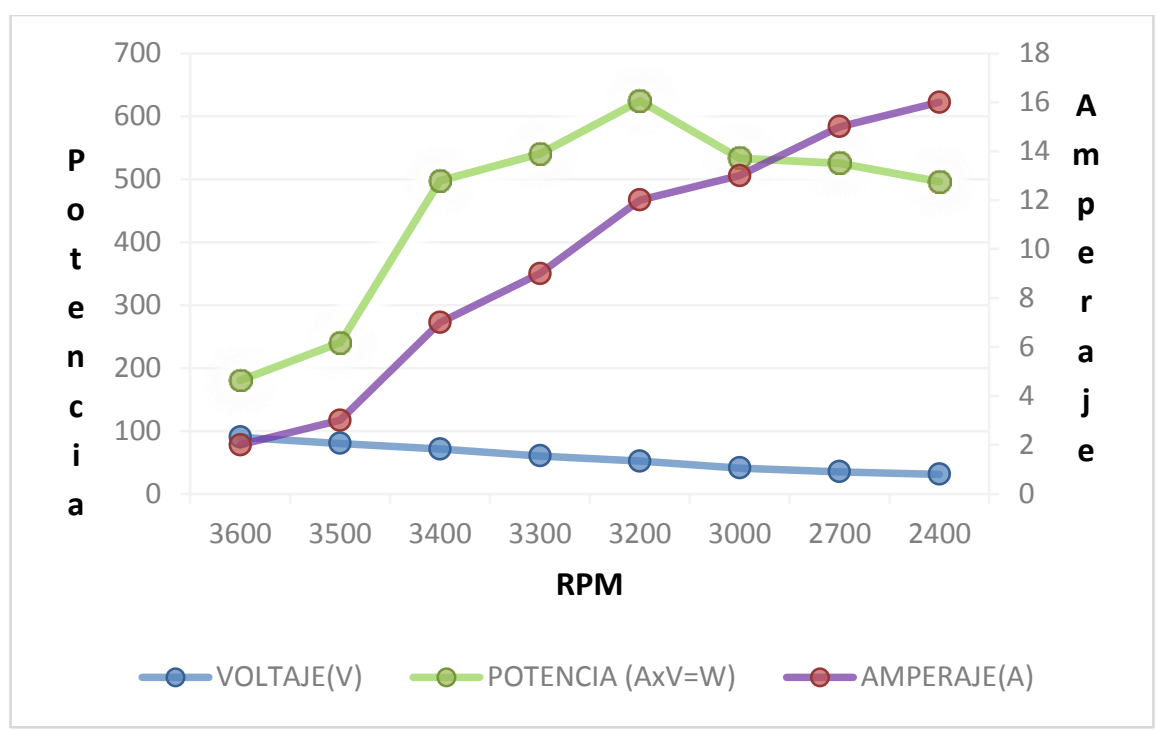

Figura 2. Resultados de prueba del motor monocilindro sin ingreso de $\mathrm{H}_{2}$ en el dinamómetro.

Se observa que la potencia al incrementarse hasta 3.781 HP (628 W) a 3200 rpm, se desarrolla de manera desordenada sin mantener un régimen de aumento de potencia estable y desciende la potencia de manera más estable a partir de la potencia máxima con referencia a cuando asciende. Posteriormente se adicionó hidróxido de sodio al 50\% en el depósito de electrolito, el compuesto al ingresar a la celda de hidrógeno rompe las moléculas de agua acelerando el proceso de obtención de hidrógeno a volumen constante; el volumen obtenido se inyectó dentro del carburador del motor monocilindro para que ingrese y se mezcle con la gasolina. Los resultados de potencia de la mezcla se indican en la Tabla 4.

Tabla 4. Resultados de pruebas del motor monocilindro con ingreso de $\mathrm{H}_{2}$ en el dinamómetro.

\begin{tabular}{|c|c|c|c|c|c|}
\hline $\begin{array}{c}\text { Nivel de } \\
\text { carga }\end{array}$ & RPM & $\begin{array}{c}\text { Voltaje } \\
\text { (V) }\end{array}$ & $\begin{array}{c}\text { Amperaje } \\
(\mathbf{A})\end{array}$ & $\begin{array}{c}\text { Potencia } \\
\text { (A.V=W) }\end{array}$ & $\begin{array}{c}\text { Potencia } \\
\text { (HP) }\end{array}$ \\
\hline 1 & 3600 & 100 & 2 & 200 & 1.212 \\
\hline 2 & 3500 & 91 & 4 & 364 & 2.205 \\
\hline 3 & 3400 & 85 & 6 & 510 & 3.090 \\
\hline 4 & 3300 & 70 & 11 & 770 & 4.665 \\
\hline 5 & 3200 & 67 & 10 & 670 & 4.060 \\
\hline 6 & 3000 & 58 & 11 & 638 & 3.866 \\
\hline 7 & 2700 & 47 & 12.5 & 587.5 & 3.560 \\
\hline 8 & 2400 & 39 & 14 & 546 & 3.308 \\
\hline
\end{tabular}

La potencia máxima cuando se adiciona hidrógeno es de 4.665 HP (770 W) a 3300 rpm y un nivel de carga de 4; este dato muestra un punto muy importante, ya que necesita más revoluciones (rpm) para alcanzar la potencia máxima y a partir de ese valor comienza a descender, sin hidrógeno la potencia máxima alcanza $3200 \mathrm{rpm}$ con una potencia $3.781 \mathrm{HP}$. El hidrógeno permite mantener un incremento de potencia de manera más estable que sin el mismo. En la Figura 3 que se muestra el cambio de la potencia de acuerdo con las cargas dadas al motor monocilindro hasta que se estabiliza. 


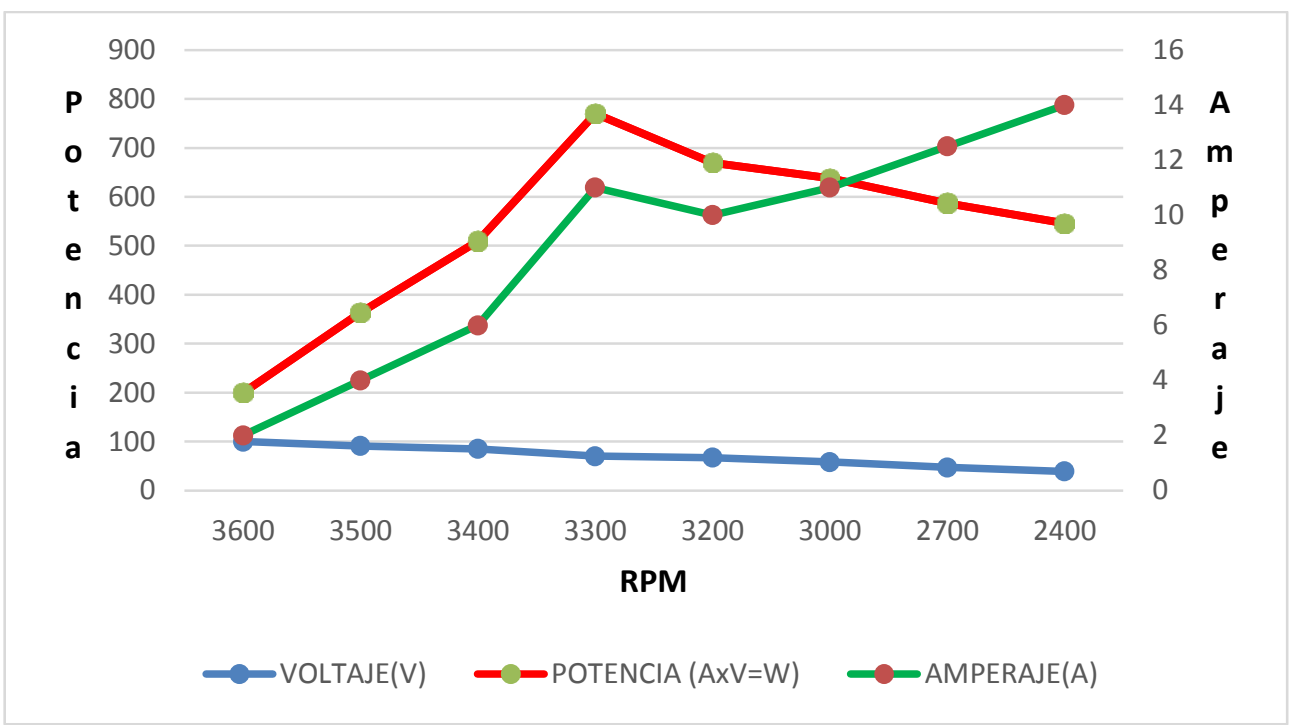

Figura 3. Resultados de prueba del motor monocilindro con ingreso de $\mathrm{H}_{2}$ en el dinamómetro.

Con el ingreso del hidrógeno el incremento de potencia se produce de manera más uniforme que sin él, esto se debe al poder calorífico que tiene este combustible lo cual reduce las fluctuaciones en los valores y permite que se estabilice el motor rápidamente de acuerdo con las cargas ingresadas al mismo.

En la Tabla 5 se muestra la diferencia de potencia teórica y real con la mezcla gasolina y aire en el motor mono cilindro con su respectivo porcentaje de variación respecto al real, también se indica el porcentaje de rendimiento de la potencia teórica y la real respecto a la potencia nominal dada por el fabricante del motor.

Tabla 5. Comparación entre la potencia teórica y real del motor monocilindro (gasolina + aire).

\begin{tabular}{|c|c|c|c|}
\hline & $\begin{array}{c}\text { Potencia } \\
\text { (HP) }\end{array}$ & $\begin{array}{c}\text { Variación } \\
(\%)\end{array}$ & $\begin{array}{c}\text { Rendimiento } \\
(\%)\end{array}$ \\
\hline Real (fabricante) & 3.78 & $3.4 \%$ & $68 \%$ \\
\hline Teórica & 3.91 & - & $71 \%$ \\
\hline
\end{tabular}

En la Tabla 6 se muestra el porcentaje de variación de la potencia teórica y real con la mezcla de gasolina y aire en el motor monocilindro. Además, se indica el porcentaje de rendimiento de la potencia teórica y la real respecto a la potencia nominal dada por el fabricante del motor.

Tabla 6. Comparación entre la potencia teórica y real del motor monocilindro (gasolina + hidrógeno).

\begin{tabular}{|c|c|c|c|}
\hline & $\begin{array}{c}\text { Potencia } \\
\text { (HP) }\end{array}$ & $\begin{array}{c}\text { Variación } \\
(\%)\end{array}$ & $\begin{array}{c}\text { Rendimiento } \\
(\%)\end{array}$ \\
\hline Real (fabricante) & 4.66 & $14.5 \%$ & $84.7 \%$ \\
\hline Teórica & 5.40 & - & $98.1 \%$ \\
\hline
\end{tabular}

Se observa en las tablas 3 y 4 que la potencia real obtenida por el motor monocilindro en el banco de pruebas del freno dinamómetro se encuentra muy aproximado al valor calculado teóricamente con una diferencia de $0.11 \mathrm{HP}$. Se interpreta que el valor se aproximó a los datos teóricos referentes a la temperatura de $27^{\circ} \mathrm{C}$ al momento de realizar las pruebas en el dinamómetro y 
calculadas con el sistema de ecuaciones bajo las condiciones de la altura de la ciudad de Quito (2850 msnm) y con la presión atmosférica de 72.794 kPa.

Al adicionar hidrógeno al combustible se obtiene un incremento de potencia de $0.74 \mathrm{HP}$, el cual es un valor significativo en comparación con la potencia teórica calculada utilizando el sistema de ecuaciones. Existe una variación entre los datos calculados, pero no se alejan significativamente de la realidad, esto se debe a que el hidrógeno tiende a fugar fácilmente debido a su densidad, controlarlo en un sistema hermético resulta muy difícil por lo cual existen pérdidas en el momento del ingreso a la cámara de combustión.

Comparando los resultados de la potencia real de la mezcla aire-gasolina con la mezcla hidrógeno-gasolina respecto a la potencia nominal dada por el fabricante de $5.5 \mathrm{HP}$, se observa un incremento de rendimiento del $16.7 \%$. En la potencia teórica obtenida comparando los resultados de la mezcla aire-gasolina e hidrógeno-gasolina respecto a la potencia nominal de 5.5 HP se observa un aumento de rendimiento del $27.1 \%$.

\section{Conclusiones y Recomendaciones}

La celda de hidrógeno produce un volumen constante de $108 \mathrm{ml} / \mathrm{min}$.

La potencia especificada por el fabricante es $5.5 \mathrm{HP}$, este valor disminuye mientras se incrementa la altura de ubicación del motor monocilindro respecto al nivel del mar.

La potencia teórica del motor monocilindro sin el ingreso de hidrógeno es $3.91 \mathrm{HP}$ y medido en el dinamómetro bajo las mismas condiciones es 3.78 HP.

La potencia teórica del motor monocilindro con ingreso de hidrógeno es $5.41 \mathrm{HP}$ y medido en el dinamómetro bajo las mismas condiciones es 4.66 HP.

Bajo las mismas condiciones de temperatura y presión atmosférica que fueron calculados y sometidos, la potencia real respecto a la potencia nominal dada por el fabricante aumenta cuando se adiciona hidrógeno en un $16.7 \%$ y en los valores teóricos calculados respecto a la potencia nominal aumenta en un $27.1 \%$ al adicionar hidrógeno a la gasolina.

El aumento del poder calorífico es $47892.4178 \mathrm{~kJ} / \mathrm{kmol}$ con el ingreso de hidrógeno y 44426.635 $\mathrm{kJ} / \mathrm{kmol}$ sin el ingreso del mismo.

Se incrementó el rendimiento térmico de 0.5460 sin el ingreso de hidrógeno y de 0.55752 con el ingreso de hidrógeno, lo cual significa que la energía es utilizada con mayor eficiencia.

Los valores de consumo calculado de gasolina-hidrógeno de $373.52 \mathrm{~g} / \mathrm{kWh}$ comparado con el valor dado por el fabricante de $395 \mathrm{~g} / \mathrm{kWh}$ (valor a altitud de $0 \mathrm{msnm}$ ) es menor, y el valor 
calculado de la mezcla gasolina-aire de $401.61 \mathrm{~g} / \mathrm{kWh}$, aumenta respecto al del fabricante. El dato del fabricante está dado a 0 msnm y valor calculado es a 2850 msnm.

Se debe realizar un estudio de uso de hidrógeno en motores diésel con variaciones en el ingreso del volumen de hidrógeno y su comportamiento a diferente altitud y régimen de giro.

Es necesario un estudio de optimización de la producción de hidrógeno controlado de manera electrónica con el uso de caudalímetros de alta precisión que estén en función al tipo de motor y sus condiciones de funcionamiento.

La temperatura de explosión dentro del cilindro es difícil de obtener, por lo cual debe realizar un estudio para el diseño de un termómetro resistente a altas presiones, temperaturas y con buena tolerancia para obtener valores experimentales reales.

Se debe realizar un estudio de las emisiones de gases emitidas por los motores que funcionan con gasolina e hidrógeno y determinar el consumo de combustible y el \% y ppm de los gases emitidos a la atmosfera.

\section{Bibliografía}

Bosch . (2005). Manual de la Técnica del Automóvil (4ta Ed.). Alemania: Bosch, 2005.

Cengel, Y. (2012). Transferencia de calor y masa (7ma Ed). México: McGrawHill.

Engel, T. (2006). Química Física. Madrid: Pearson Educación S.A.

Payri, F. (2011). Motores de Combustión Interna Alternativos. Barcelona: Reverté.

Águeda, E. (2009). Fundamentos Tecnológicos del Automóvil (1ra Ed.). Madrid: Thomson

Editores.

Gutiérrez, L. (2005). El hidrógeno, combustible del futuro. Rev.R.Acad.Cienc.Exact.Fís.Nat. (Esp). V Programa de Promoción de la Cultura Científica y Tecnológica. Vol. 99, №. 1, pp 49-67.

Fàbrega Ramos, Marc. (2009) Hidrógeno: Aplicación en motores de combustión interna. Universitat Politècnica de Catalunya. Departament de Ciència i Enginyeria Nàutiques. Cataluña, España.

Casaravilla, G. (2012). Hacia un transporte automotor racional y eficiente: Autos Híbridos y Eléctricos. Montevideo, Uruguay. Proyecto de la Universidad de la República y Facultad de Ingeniería.

Familiar Xaudaró, Crístian. (2011). Inyección de hidrógeno como potencial mejora de los motores actuales. Universitat Politècnica de Catalunya. Departament de Ciència i Enginyeria Nàutiques. Cataluña, España. 
Sáiz Casa, David. (2011). Adaptación de un motor de combustión interna alternativo de gasolina para su funcionamiento con hidrógeno como combustible. Aplicaciones energética y de automoción. Universidad Pública de Navarra. España.

Velástegui, S. Jesús, C. Torres, C \& Erazo, E. (2015). Obtención de los parámetros característicos del motor Daewoo 1800cc a gasolina con inyección directa de hidrógeno. Universidad de las Fuerzas Armadas ESPE. Departamento de Ciencias de la Energía y Mecánica. Latacunga, Ecuador.

Bennaceur, K. Clark, B. Orr, F. Ramakrishnan, T. \& Stout, E. (2005). El hidrógeno: ¿Un futuro portador energético?. Obtenido de https://www.slb.com/ /media/Files/resources/oilfield_review/ spanish05/sum05/p34_47.pdf 